\title{
Spherical mirror testing by phase retrieval wavefront sensor
}

\author{
Xinxue Ma*and Jianli Wang \\ Changchun Institute of Optics, Fine Mechanics and Physics, Chinese Academy of Sciences, \\ Changchun 130033, China \\ *Corresponding author: xinxuema@hotmail.com
}

\begin{abstract}
In order to verify the estimated wavefront ability of the phase retrieval wavefront sensor (PRWS), a measured spherical mirror of experiment platform was set up with the method of PRWS. PRWS technology is based on the focal plane image information wavefront solver in the focal plane wavefront measured technology, whose principle is sampling a number of the given defocus images; get the wavefront phase information by solving the optical system wavefront with Fourier optical diffractive theory and mathematics optimization. In order to validate the veracity of PRWS, both the PRWS measurement results and ZYGO interferometer measurement results were compared; experimental results demonstrate that agreement is obtained among the errors distribution, PV value and RMS value of ZYGO interferometer, so PRWS technology can effectively estimate the aberrations of spherical mirror.
\end{abstract}

Key words: Phase retrieval; Wavefront sensor; Spherical mirror; Zernike polynomial; Aberration

\section{Introduction}

In the machining processing of large-scale optics mirror in situ real-time estimation and alignment with the use of the optical system during dynamic measurement of wave aberrations is difficult to accomplish for the present tradition optical inspection equipment ${ }^{[1-3]}$. In order to control the optical quality of the telescope, we need a simple and with high accuracy method. This article proposed phase retrieval wavefront sensor (PRWS) technology ${ }^{[4-8]}$ based on the focal plane image information wavefront solver in the focal plane estimated wavefront technology, whose principle is given by sampling a number of the defocus images ${ }^{[9-14]}$; solve the optical system wavefront by Fourier optical methods. System hardware is simple, free from the environment (especially the vibration) influence of optical components and systems for dynamic estimation, real-time display of measurements ${ }^{[7,15]}$, which has good application prospects in the field of the optical processing, system alignment, active optics, adapt optics et al.

In order to verify the estimated wavefront ability of PRWS, this paper set up a measured spherical mirror of experiment platform with the method of PRWS based on phase retrieval (PR) theory research and experimental verification. This paper compared PRWS measurement results with ZYGO interferometer ${ }^{[16-22]}$ measurement results, experimental results demonstrate that agreement is obtained among the errors distribution, PV value and RMS value of ZYGO interferometer, so using PRWS technology can effectively test the spherical mirror aberration, which illustrates the feasibility and accuracy of PRWS measurement methods.

This paper is organized as follows: the theory of PRWS is presented in section 2, the design of experiment in section 3 and the summary in section 4 .

\section{The principles of PRWS}

Phase retrieval (PR) system is the wavefront detector of a focal plane waves; a laser spot light on the object plane is a target designated from the focal plane image acquisition, use the acquired image, the defocus of the corresponding image, known pupil size and shape to reverse solve the aberration of the optical system ${ }^{[23]}$. The structure of the PR system is shown in Fig. 1. 


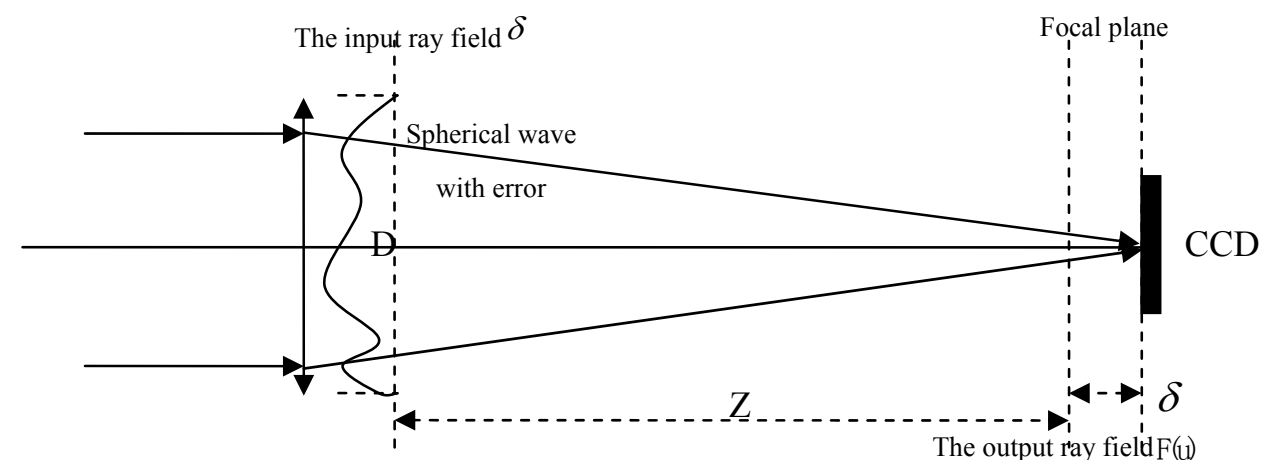

Fig.1. Schematic of optical path of PR

Assuming that the aperture of a measured optical system is $D$, the focal length is $Z$, the center wavelength of the laser light source is $\lambda$, whose pupil constraint function is $|f(x)|$, the generalized pupil function for focus plane is

$f(x)=|f(x)| \exp [i \theta(x)]$,

where $\theta$ is wavefront distortion and can be obtained with Zernike polynomial fitting: $\theta(x)=\sum_{n} \alpha_{n} Z_{n}(x)$, the real number ${ }^{\alpha}{ }_{n}$ represents the first $n^{\text {th }}$ terms of polynomial coefficients, ${ }_{n}$ indicates the first $n^{\text {th }}$ terms of Zernike polynomials basement.

For linear optical system, when the generalized pupil $f(x)$ whose defocus is $\delta$ in the plane, the impulse response function $F(u)$ is $F(u)=|F(u)| \exp [i \psi(u)]=\mathcal{F}\{f(x) \exp [\varepsilon(x, \delta)]\}$,

where $x$ is the coordinates of the pupil domain, $u$ is the coordinates of the image domain, both of them are two-dimensional vector field coordinates. $\psi_{\text {is }}$ the phase part of the impulse response, $\mathcal{F}$ is two-dimensional Fourier transform, $\varepsilon(x, \delta)$ is wavefront aberration caused by defocus $\delta$ in the position $x$.

For a PR system, $|f(x)|$ of equation (1) is the priori conditions of a known optical system, corresponds to the size and shape of the pupil. $|F(x)|_{\text {is }}^{2}$ the image collected by CCD where the defocus is $\delta$. Therefore, the purpose that we estimate wavefront by PR is to get $\alpha_{n}$ by the above known quantity. So formal description of the problem for: $\left|f(x), \delta_{1},\right| F_{1}\left(\left.u\right|^{2}, \delta_{2}, \mid F_{2}\left(\left.u\right|^{2}, \ldots, \delta_{M},\left|F_{M}(u)\right|^{2}\right.\right.$ are known. Image acquisition distance from the focal plane at $\delta, \delta_{2}, \ldots, \delta_{M}$ respectively is $\mid F_{1}\left(\left.u\right|^{2}, \mid F_{2}\left(\left.u\right|^{2}, \ldots, \mid F_{M}\left(\left.u\right|^{2}\right.\right.\right.$.

The objective function and the partial derivative of PR objective function with respect to ${ }_{n}$ respectively is formula (3) and formula (4)

$$
\begin{aligned}
& B_{k}=E_{F k}^{2}=N^{-2} \sum_{m=1}^{M} \sum_{u}\left[\left|G_{m, k}(u)\right|-|F(u)|\right]^{2}, \\
& \partial_{a_{n}} B_{k}=-2 \sum_{m} \sum_{x}|f(x)|\left|g_{m, k}(x)\right| \sin \left[\theta_{m, k}{ }^{\prime}(x)-\theta_{m, k}(x)\right] Z_{n}(x) .
\end{aligned}
$$


With the objective function (3) and its impact on the Zernike coefficient derivative (4), we can use the mathematical optimization algorithm to solve various Zernike wavefront coefficient values, here we use LBFGS algorithm that the phase diversity (PD) ${ }^{[24-30]}$ experiment has been able to solve.

\section{The design of the experiments}

\section{A. Experimental theory and components}

The schematic diagram of PRWS is shown in Fig. 2. Gaussian beam emitted from the laser through the pinhole into a spherical wave, passes through the lens 2 into a parallel light, the light projects in the prism through the prism is divided into two parts, a part need not be considered, another part of the parallel reflects after a light through the lens 1 converge after the measured mirror, the reflected beam with phase ${ }^{[31,32]}$ information (i.e. aberration), again divided into two groups by a beam splitter, one part backtrack, the other part through the converging lens 3 converged at CCD camera, which is placed on a movable platform, move along the optical axis and the angle of the camera posture fine-tuning to get the focus before and after receiving a different amount of defocus images ${ }^{[33-35]}$, used in realizing the estimated wavefront based on the PR. We can obtain aberration of the measured spherical mirror with the PR algorithm.

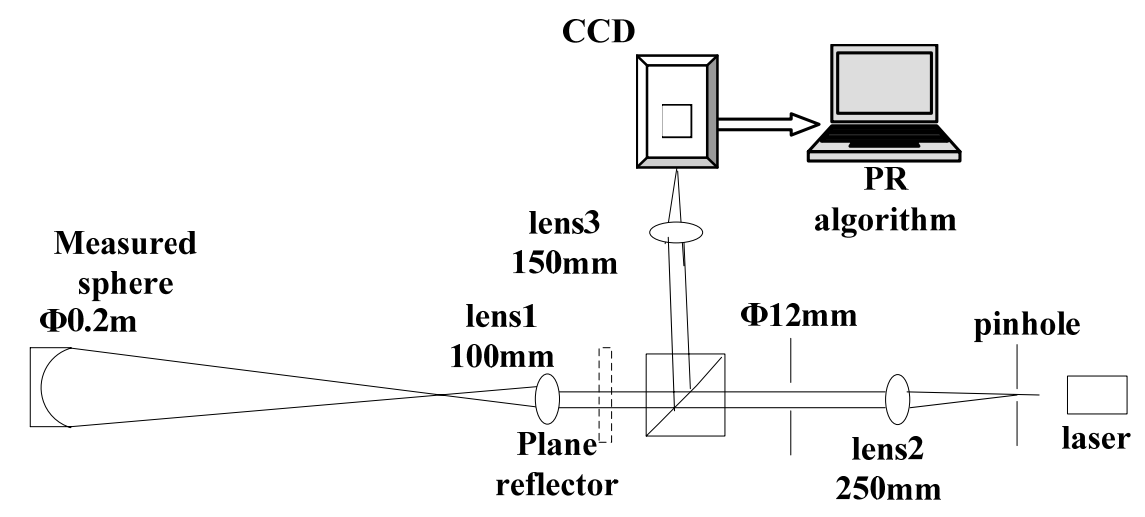

Fig.2 Schematic diagram of PRWS

The focal length of measured spherical mirror is $0.2 \mathrm{~m}$, the center wavelength is $632.5 \mathrm{~nm}$, focal length of 3 in the experimental system is $0.15 \mathrm{~m}$, the exit pupil caliber is $0.012 \mathrm{~m}$, and thedepth of focus is about $0.286 \mathrm{~mm}$. In the experiment, the defocus we select is $0 \mathrm{~mm}, \pm 1 \mathrm{~mm}, \pm 1.5 \mathrm{~mm}, \pm 2 \mathrm{~mm}$. Camera pixel size is $6.45 \mu \mathrm{m}$, each defocus position respectively intercept $128 * 128$ pixel size of target region, the exposure time is $20 \mathrm{~ms}$, the accuracy of mobile platform is $\pm 5 \mu \mathrm{m}$. The experimental optical path is shown in Fig.3.

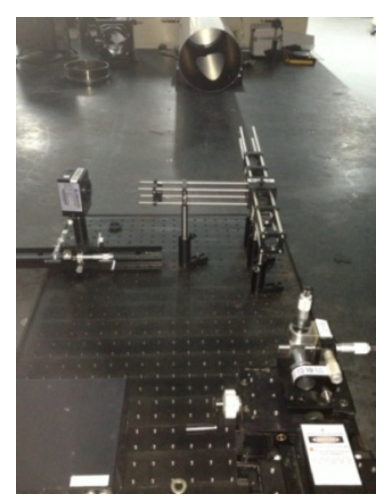

Fig.3 The experimental system of PRWS

\section{B. Experimental procedures}


In the process of the entire experimental, we not only prove the estimation ability of PRWS, but also prove the accuracy of PRWS, therefore during the experimental design, in order to ensure that the position of the measured spherical mirror during the whole experiment is invariable, we need to find the good distance between spherical mirrorwith PRWS devices and between spherical mirror with ZYGO, respectively, based on the focal lengths of the measured spherical mirror. Then separately measure the spherical mirror with PRWS and ZYGO.

Step1: build experiment system.Firstly, fix the $0.2 \mathrm{~m}$ telescope, as shown in Fig. 4; secondly, place the laser and the position of the pinhole, as shown in Fig. 5. The requirements of laser position are: laser light and the center of the telescope mirror are coaxial. After regulating, put the microscope objective and a pinhole placed in front of the laser, make the light from the pinhole is an ideal spherical wave. Lastly, reconfirm the laser, pinhole, spherical mirror are coaxial, as shown in Fig. 6.

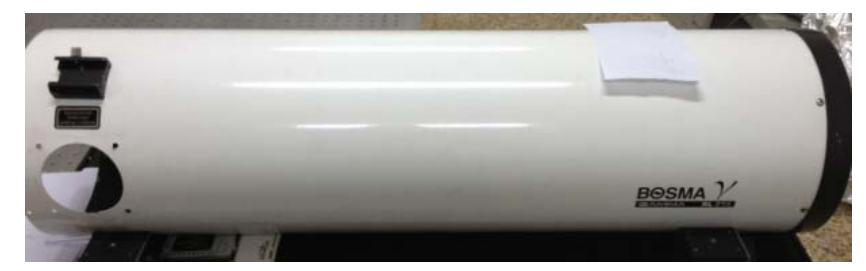

Fig.4 Tested telescope

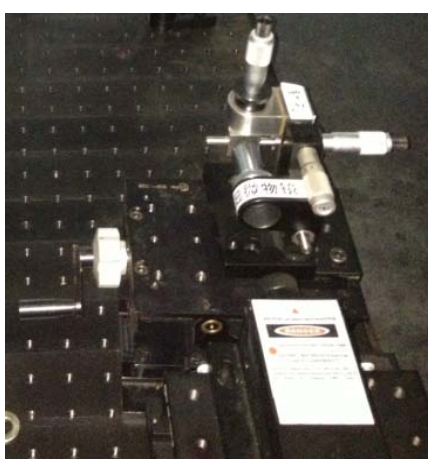

Fig.5 laser and pinhole

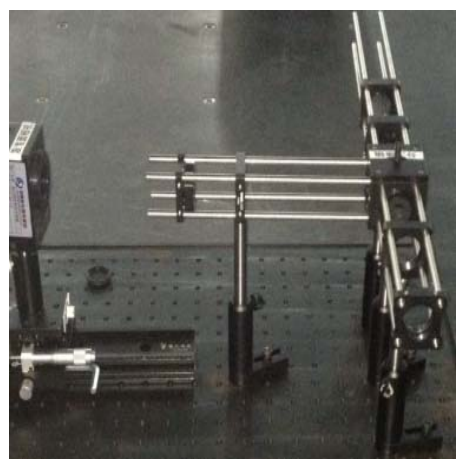

Fig.6 Coaxial system

Step 2: add the lens 1, and adjust the position of the lens 1 to make it be coaxial with the laser and pinhole until the spherical wave emitted from the pinhole through the lens 1 into parallel light;

Step 3: add the splitting prism, light bar and the lens 2 in the optical path, and make them be coaxial with the measured spherical mirror and pinhole, then according to the parallelism of the return light beam from splitter to adjust the position of the lens 2;

Step 4: adjust the position of the lens 3 and the camera to make the light beam reflect from the measured spherical light through the prism to enter into the camera;

Step 5: after adjusting the system, adjust the translation stage to make the point of light least, then record the location and use it as the position of the focal point;

Step 6: capture images with the camera at the position of the defocus distances of $\pm 1 \mathrm{~mm}, \pm 1.5 \mathrm{~mm}$, $\pm 2 \mathrm{~mm}$

Step 7: put the plane mirror between the lens 1 and the prism, repeat the above steps to capture images with the camera;

Step 8: dispose the images collected of the sixth and seventh steps with the PD algorithm, get the aberration of the measured spherical mirror;

Step 9: ensure that the position of the measured spherical mirror remains unchanged, use the ZYGO 
interferometer estimate the measured spherical mirror. Figure 7 is a diagram of the estimation experiment with the ZYGO interferometer.

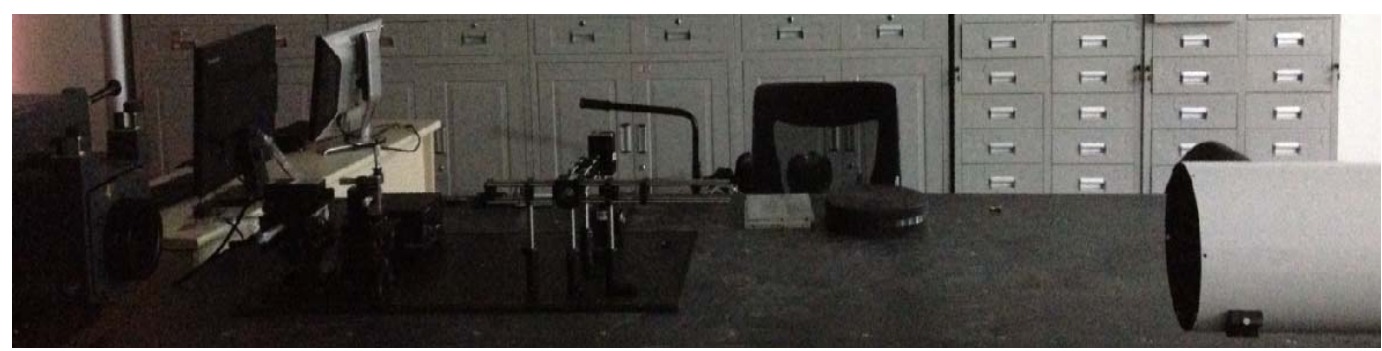

Fig.7 The experimental system of ZYGO interferometer measurement

\section{Experimental results and discussion}

We dispose the collected seven images with the PR algorithm, obtain the measured result of the spherical mirror is shown in Fig.8. The measured result with the ZYGO interferometer is shown in Fig. 9.

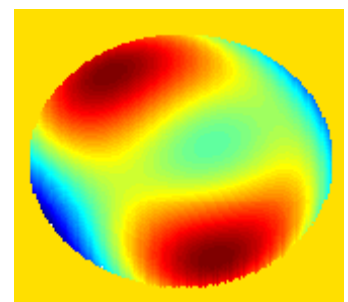

Fig. 8 Result of PRWS, RMS $=0.272 \lambda, \quad P V=1.608 \lambda$

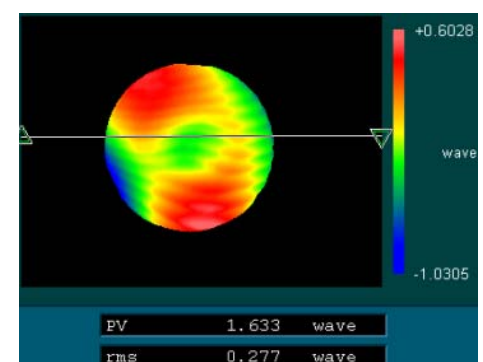

Fig.9 Result of ZYGO interferometer, RMS $=0.277 \lambda, \quad P V=1.633 \lambda$

In order to illustrate the accuracy and viability of the PRWS better, we rotated the spherical mirror a definite degree, and then estimate the spherical mirror with steps one to nine, the obtained measurement results are shown in Fig.10 and Fig. 11.

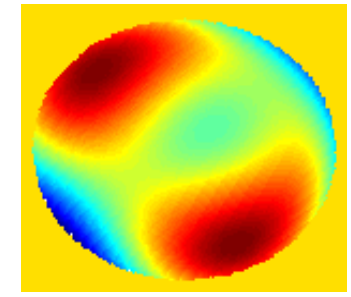

Fig.10 Result of PRWS after rotation,

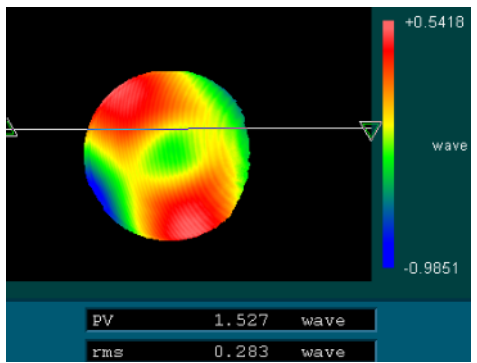

Fig.11 Result of ZYGO interferometer after rotation, $\mathrm{RMS}=0.283 \lambda, \quad \mathrm{PV}=1.527 \lambda$

In order to illustrate the accuracy and repeatability of PR, we rotated the spherical mirror some degree, and then estimate the spherical mirror with steps one to nine, the obtained measurement results are shown in Fig. 12.

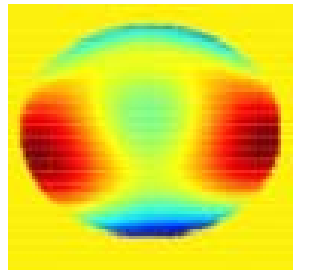

Fig. 12 Result of PR measurement after rotation, RMS $=0.271 \lambda, \quad P V=1.659 \lambda$ 
In order to verify the accuracy of PRWS we compared the 19th Zernike polynomials of Fig.10 and Fig.11 and get the results are shown in table 1.

Table.1 comparison of Zernike polynomials

\begin{tabular}{|c|c|c|c|}
\hline ITEMS & Zernike & ZYGO & PRWS \\
\hline 1 & Piston & 0 & 0 \\
\hline 2 & $\mathrm{X}$ tilt & 0 & 0 \\
\hline 3 & Y tilt & 0 & 0 \\
\hline 4 & Defocus & 0 & 0 \\
\hline 5 & Ast $\mathrm{x}$ & -0.380 & -0.372 \\
\hline 6 & Ast Y & -0.552 & -0.543 \\
\hline 7 & Coma $\mathrm{x}$ & 0.018 & -0.017 \\
\hline 8 & Coma Y & 0.044 & 0.043 \\
\hline 9 & Primary Spherical & -0.217 & -0.213 \\
\hline 10 & Trefoil $\mathrm{x}$ & -0.013 & -0.012 \\
\hline 11 & Trefoil y & 0.240 & 0.235 \\
\hline 12 & Secondary Ast $\mathrm{x}$ & 0.045 & 0.044 \\
\hline 13 & Secondary Ast y & 0.028 & 0.027 \\
\hline 14 & Secondary coma $\mathrm{x}$ & -0.065 & -0.063 \\
\hline 15 & Secondary coma y & -0.007 & -0.006 \\
\hline 16 & Secondary Spherical & 0.100 & 0.099 \\
\hline 17 & Tetrafoil x & 0.030 & 0.029 \\
\hline 18 & Tetrafoil y & 0.014 & 0.013 \\
\hline 19 & Secondary Trefoil $\mathrm{x}$ & -0.020 & -0.019 \\
\hline
\end{tabular}

From the results of table 1, we can see that the Zernike polynomials of PRWS and ZYGO are linear relationship, which is the same as the difference RMS between PRWS and ZYGO. From the measurement result after rotating, as shown in Fig.12, the results of estimated wavefront before and after rotation are agreement, which verifies the repeatability and effectiveness of PRWS measurement. Seeing from Fig. 8 and Fig.9, Fig. 10 and Fig. 11, there is an agreement among the errors distribution, PV value and RMS value of ZYGO interferometer, which explains the feasibility and accuracy of the PRWS measurement methods.

\section{Conclusions}

This paper set up an estimation spherical mirror of experiment platform with the method of PRWS, from the contrast of the measurement results before and after rotation, which verified the PRWS measurement repeatability and effectiveness. In order to validate the veracity of PRWS, this paper compared PRWS measurement results with ZYGO interferometer measurement results, experimental results demonstrate that agreement is obtained among the errors distribution, PV value and RMS value of ZYGO interferometer, so using PRWS technology can effectively estimate the spherical mirror aberration, which explains the feasibility and accuracy of the PRWS measurement methods, which provides the feasibility to data support for our later search.

\section{References}


1. Brown B, Aaron M (2001) The politics of nature. In: Smith J (ed) The rise of modern genomics, 3rd edn. Wiley, New York.

2. BRADY GREGORY R, FIENUP J R. Improved optical metrology using phase retrieval[C].Optical Fabrication \& Testing. Rochester, NY, 2004, 10 1-3.

3. Yang Huizhen, Gong Chenglong. Phase retrieval for a kind of wavefront sensor based on pupil phase diversity [J]. Acta Optica Sinica, 2011, 31(11):1112002.

4. Osten W. Some answers to new challenges in optical metrology[C].Proc. SPIE, 2008, 7155 715503-1 715503-16.

5. OHARA C M, FAUST J A, LOWMAN A E, et al. Phase retrieval camera optical testing of the advanced mirror system demonstrator [C].SPIE, 2004, 5487 1744-1756.

6. GERCHBERG R W, SAXTON W O. A practical algorithm for the determination of phase from image and diffraction phase pictures [J]. Optic. 1972, 35(2) 237-246.

7.FIENUP J R. Phase retrieval algorithms: A comparison [J]. Appl Opt. 1982, 21(15) 2758-2769.

8. FIENUP J R, MARRON J C, SCHULZ T J et al. Hubble space telescope characterized by using phase-retrieval algorithms [J]. Appl Opt.1993, 32(10) 1747-1767.

9. DEAN BRUCE H, ARONSTEIN DAVID L, SMITH J SCOTT, et al. Phase retrieval algorithm for JWST flight and testbed telescope[C].Proc. of SPIE, 2006, 6265 1-17.

10. Li Fei, Rao Changhui. Study on phase diversity wavefront sensor [J]. Acta Optica Sinica, 2011, 31(8):0804001

11. Liang Shitong, Yang Jianfeng, Xue Bin. A new phase diversity wave-front error sensing method based on genetic algorithm [J]. Acta Optica Sinica, 2010, 30(4):1015-1019.

12. Jiang Pengzhi, Ma Haotong, Zou Yongchao et al. Study of aberration correction in light path of adaptive optical system [J].Acta Optica Sinica, 2011, 31(12):1214002.

13. DEVANEY A J, CHILDLAW R. On the uniqueness question in the problem of phase retrieval from intensity measurement [J]. JOSA A, 1978, 68(10) 1352-1354.

14. Han Bing, Xiao Wen, Pan Feng et al. Optimization of Space Sampling Distance of Phase Retrieval Algorithm for In-Line Digital Holography [J].Laser\&Optoelectronics Progress, 2012, 49,120903.

15. Fu Fuxing, Zhang Bin.Recovery of High Frequency Phase of Laser Beam with Wavefront Distortio[J].Chinese J,Lasers,2011,38(4),0402009.

16. BRADY GREGORY R, FIENUP J R. Phase retrieval as an optical metrology tool[C] Optical Fabrication \& Testing. Topical Meeting of the Optical Society of America, SPIE Technical Digest 2005, TD03 139-141.

17. Millerd J E, Wyant J C. Simultaneous phase-shifting Fizeau interferometer [P].US Patent 2005, 20050046864

18. Leslie Deck. Vibration-resistant phase-shifting interferometry[J]. Applied Optics, 1996, 35(34) 6655-6662.

19. Burge J H, Wyant J C. Applications of computer-generated holograms for interferometric measurement of large aspheric optics[C].Proc.SPIE. 1995, 2576 258-269.

20. Reichelt S, Pruss C, Tiziani H J. Absolute interferometric test of aspheres by use of twin computer- generated holograms [J]. Applied Optics. 2003, 42(22) 4468-4479.

21. Sommargren G E, Phillion D W, Campbell E W. Sub-nanometer interferometry for aspheric mirror fabrication[C].The 9th International Conference on Production Engineering, Osaka, Japam. 1999

22. Reichelt S,Tiziani H J. Twin-CGHs for absolute calibration in wavefront testing interferometry [J]. Optics Communications. 2003, 220 23-32.

23. MA Xinxue, WANG Jianli, WANG Bin. Study on phase retrieval algorithm [J]. Laser \& Infrared. 2012, 42(2) 217-221.

24. WANG J L, WANG Z Y, WANG B, et al. Image restoration by Phase-diversity Speckle [J]. Opt. Precision Eng. 2011, 19 (5):1165-1170. 
25. WANG B, WANG Z Y, WANG J L, et al. Phase-diverse speckle imaging with two cameras [J]. Opt. Precision Eng. 2011, 19(6): 1384-1390.

26.ZHAO J Y,CHEN Z F,WANG B, et al. Improvement of Phase Diversity Object Function's Parallelity J. Opt. Precision Eng. 2012, 20(2) : 431-438.

27. WANG B,MA XX, et al. Calibration of no-common path aberration in AO system using multi-channel phase-diversity wave-front sensing [J]. Opt. Precision Eng. 2013, 21(7): 1683-1692.

28. WANG ZY, WANG B, WU YH, et al. Calibration of non-common path static aberrations by using phase diversity technology [J] .Acta Optica Sinica, 2012, 32(7):0701007.

29. ZHAO J Y, MA XX, et al. Image restoration based on real time wave-front information [J]. Opt. Precision Eng. 2012, 20 (6):1350-1356.

30. R. H. Byrd, P. Lu, and J. Nocedal, “A limited-memory algorithm for bound-constrained optimization,”SIAM Journal on Scientific and Statistical Computing, 1995, 16(5), pp. 1190 - 1208.

31. Min Gu, Hongchun Bao, Xiaosong Gan, et al. Tweezing and manipulating micro- and nanoparticles by optical nonlinear endoscopy. [J]. Light:Science\&Applications(2014)3, e126.

32. Nicolo` Accanto, Jana B Nieder, et al. Phase control of femtosecond pulses on the nanoscaleusing second harmonic nanoparticles[J]. Light:Science\&Applications (2014)3, e143.

33. Lin Li, Wei Guo, Yinzhou Yan, et al. Label-free super- resolution imaging of adenoviruses by submerged microsphere optical nanoscopy. [J]. Light:Science\&Applications(2013)2, e104.

34. Arif E Cetin, Ahmet F Coskun, Betty C Galarreta, et al. Handheld high-throughput plasmonic biosensor using computational on-chip imaging. [J]. Light:Science\&Applications(2014)3, e122.

35. Xosé Luís Deán-Ben and Daniel Razansky . Adding fifth dimension to optoacoustic imaging: volumetric time-resolved spectrally enriched tomography [J]. Light:Science\&Applications (2014)3,e137. 